\title{
On the Resistance-Harary Index of Graphs Given Cut Edges
}

\author{
Hongzhuan Wang, Hongbo Hua, Libing Zhang, and Shu Wen \\ Huaiyin Institute of Technology, Faculty of Mathematics and Physics, Huaian, Jiangsu 223003, China \\ Correspondence should be addressed to Hongzhuan Wang; wanghz412@163.com
}

Received 22 June 2017; Accepted 26 November 2017; Published 21 December 2017

Academic Editor: Robert Zaleśny

Copyright (C) 2017 Hongzhuan Wang et al. This is an open access article distributed under the Creative Commons Attribution License, which permits unrestricted use, distribution, and reproduction in any medium, provided the original work is properly cited.

Graphs are often used to describe the structure of compounds and drugs. Each vertex in the graph represents the molecule and each edge represents the bond between the atoms. The resistance distance between any two vertices is equal to the resistance between the two points of an electrical network. The Resistance-Harary index is defined as the sum of reciprocals of resistance distances between all pairs of vertices. In this paper, the extremal graphs with maximum Resistance-Harary index are determined in connected graphs with given vertices and cut edges.

\section{Introduction}

Recently, the development of computational chemistry owes much to the theory of graphs. One of the most popular areas is topological index. The molecular topological index can describe the molecular structure quantitatively and analyze the structure and performance of molecules.

Among them, the most common topological index is resistance distance. The resistance distance is raised by Klein and Randić [1] as a distance function. Let $G$ be a simple connected graph with vertex set $V(G)$ and edge set $E(G)$.

The resistance distance between vertices $u$ and $v$ of $G$ is recorded as $r_{G}(u, v)$, which represents the effective resistance between two nodes $u$ and $v$ in an electronic network; that is to say, the vertex corresponds to the node of the electronic network, and the edge corresponds to the unit resistance.

Similar to the traditional path distance, the resistance distance not only has good mathematical characteristics but also has good physical characteristics [2,3]; at the same time, it also has a good application in chemistry.

Harary index is another kind of graph invariants proposed by Plavšić et al. [4] and by Ivanciuc et al. [5] in 1993 for the characterization of molecular graphs. Name this in honor of Professor Frank Harary's birthday. The Harary index $H(G)$ is defined as the sum of reciprocals of distances between all pairs of vertices of the graph $G$; that is,

$$
H(G)=\sum_{\{u, v\} \subseteq V(G)} \frac{1}{d(u, v)} .
$$

Gutman [6] and Xu [7] investigated the Harary index of trees; they studied the Harary index of tree and pointed out that the path and the star attain the minimal and maximal value of Harary index, respectively, among a tree with given $n$ vertices. In recent years, the Harary index was well studied in mathematical and chemical literatures [8].

The reciprocal resistance distance is also called electrical conductance, Klein and Ivanciuc [9] investigated QSAR and QSPR molecular descriptors computed from the resistance distance and electrical conductance matrices, and they proposed the global cyclicity index $C(G)$ as

$$
C(G)=\sum_{u v \in E(G)}\left[\frac{1}{r(u, v)}\right]-|E G|,
$$

where the sum is over all edges of $G$.

In [10], using graph theory, electronic networks, and real number analysis methods, Yang obtains some conclusions about the global cyclicity index.

Following the definition of the Harary index, Chen et al. [11] generalized the global cyclicity index and introduced Resistance-Harary index, defined as

$$
\mathrm{RH}(G)=\sum_{\{u, v\} \subseteq V(G)} \frac{1}{r(u, v)} .
$$

In [11], Chen et al. depicted the graphs with largest and smallest Resistance-Harary index in all unicyclic graphs. 
All graphs considered in this paper are finite and simple.

Before proceeding, we introduce some further notation and terminology. A cut edge is an edge whose deletion increases the number of components. Denote by $K_{n}, C_{n}$, and $S_{n}$ the complete, cycle, and star graph on $n$ vertices, respectively. For a graph $G$ with $v \in V(G), G-v$ denotes the graph resulting from $G$ by deleting $v$ and its incident edges. For an edge $u v$ of the graph $G$ (the complement of $G$, resp.), $G-u v(G+u v$, resp. $)$ denotes the graph resulting from $G$ by deleting (adding, resp.) $u v$.

For other definitions, we can refer to [12].

In this thesis, we consider the Resistance-Harary index of graphs given cut edge. We will determine the graphs with maximum Resistance-Harary index in connected graphs given vertices and cut edges.

\section{Some Preliminary Results}

We first list or prove some lemmas as basic but necessary preliminaries.

Lemma 1 (see $[1,13,14])$. Let $x$ be a cut vertex of a graph $G$, and let $a$ and $b$ be vertices in different components of $G-x$. Then

$$
r(a, b)=r(a, x)+r(x, b) .
$$

Lemma 2. The functions $f_{1}(x)=x /(2 / x+m)-(x-1) /(2 /(x-$ 1) $+m)$ for $x \geq 2$ and $m>0$ and $f_{2}(x)=x /(2 / x+A)(2 / x+B)$ for $x>1$ and $A>0, B>0$ are strictly increasing.

Proof. By simple calculation,

$$
\begin{aligned}
f_{1}^{\prime}(x) & =\frac{4 x+x^{2} m}{(2+m x)^{2}}-\frac{m x^{2}-2 m x+m+4 x-4}{(2+m x-m)^{2}} \\
& =\frac{4 m(2 x-1)+16}{(2+m x)^{2}(2+m x-m)^{2}}>0 .
\end{aligned}
$$

Since $x \geq 2$ and $m>0, f_{1}^{\prime}(x)$ is the derivative of function $f_{1}(x)$ on $x$. Obviously, $f_{1}(x)$ is a strictly increasing function for $x \geq 2$ and $m>0$.

Similarly, we prove that the function $f_{2}(x)=x /(2 / x+$ $A)(2 / x+B)$ for $x>1$ and $A>0, B>0$ is also an increasing function. We finally obtain the result.

Lemma 3. Let $G$ be a connected graph with at least three vertices. If $G$ is not isomorphic to $K_{n}$, Let $G^{*}=G+e$, and then $R H\left(G^{*}\right)>R H(G)$.

Proof. Suppose that $G$ is not a complete graph. Then there exists a pair of vertices $v_{i}$ and $v_{j}$ in $G$ such that $v_{i} v_{j} \in E(\bar{G})$.

Let $G^{*}=G+v_{i} v_{j}$, we have

$$
\begin{gathered}
\mathrm{RH}\left(G^{*}\right)-\mathrm{RH}(G)=\mathrm{RH}\left(G+v_{i} v_{j}\right)-\mathrm{RH}(G) \\
=\sum_{1 \leq r<s \leq n}\left(\frac{1}{r_{G^{*}}\left(v_{r}, v_{s}\right)}-\frac{1}{r_{G}\left(v_{r}, v_{s}\right)}\right)
\end{gathered}
$$

$$
\begin{aligned}
= & \left(\frac{1}{r_{G^{*}}\left(v_{i}, v_{j}\right)}-\frac{1}{r_{G}\left(v_{i}, v_{j}\right)}\right) \\
& +\sum_{\substack{1 \leq r<s \leq n \\
(r, s) \neq(i, j)}} \frac{1}{r_{G^{*}}\left(v_{r}, v_{s}\right)}-\frac{1}{r_{G}\left(v_{r}, v_{s}\right)} .
\end{aligned}
$$

We only to prove that $r_{G^{*}}\left(v_{i}, v_{j}\right)<r_{G}\left(v_{i}, v_{j}\right)$; the proof of $r_{G^{*}}\left(v_{r}, v_{s}\right)<r_{G}\left(v_{r}, v_{s}\right)$ for $(r, s) \neq(i, j)$ is similar. We distinguish the following two cases.

Case 1. $v_{i}$ and $v_{j}$ are vertices of cycle $C_{g}$ in $G$, where $g$ is the length of $C_{g}$.

Let $d_{1}\left(v_{i}, v_{j}\right)$ and $d_{2}\left(v_{i}, v_{j}\right)$ be distance between $v_{i}$ and $v_{j}$ in the cycle $C_{g}$, respectively. By the definition of resistance, we have

$$
\begin{aligned}
& \frac{1}{r_{G^{*}}\left(v_{i}, v_{j}\right)}-\frac{1}{r_{G}\left(v_{i}, v_{j}\right)} \\
& =\left(\frac{1}{d_{1}\left(v_{i}, v_{j}\right)}+\frac{1}{d_{2}\left(v_{i}, v_{j}\right)}+1\right) \\
& \quad-\left(\frac{1}{d_{1}\left(v_{i}, v_{j}\right)}+\frac{1}{d_{2}\left(v_{i}, v_{j}\right)}\right)>0 .
\end{aligned}
$$

That is to say, $r_{G^{*}}\left(v_{i}, v_{j}\right)<r_{G}\left(v_{i}, v_{j}\right)$.

Case 2. $v_{i}$ and $v_{j}$ are not vertices in any cycle of $G$.

In this case, we have

$$
\begin{gathered}
r_{G^{*}}\left(v_{i}, v_{j}\right)-r_{G}\left(v_{i}, v_{j}\right)=\frac{d_{G}\left(v_{i}, v_{j}\right)}{1+d_{G}\left(v_{i}, v_{j}\right)}-d_{G}\left(v_{i}, v_{j}\right) \\
=d_{G}\left(v_{i}, v_{j}\right)\left(\frac{1}{1+d_{G}\left(v_{i}, v_{j}\right)}-1\right)<0
\end{gathered}
$$

This completes the proof.

Lemma 4. Let $x y \in E(G)$ be a cut edge in $G$, and let $G_{1}$ and $G_{2}$ be the two components of $G-x y$. Suppose further that $x \in$ $V\left(G_{1}\right)$ and $y \in V\left(G_{2}\right)$, and then $R H(G)=R H\left(G_{1}\right)+R H\left(G_{2}\right)+$ $\sum_{a \in V\left(G_{1}\right)} \sum_{b \in V\left(G_{2}\right)}\left(1 /\left(r_{G}(a, x)+r_{G}(b, y)+1\right)\right)$.

Proof. By the definition of Resistance-Harary index and by Lemma 1, we have

$$
\begin{aligned}
\mathrm{RH}(G)= & \sum_{a, b \in V\left(G_{i}\right)} \frac{1}{r_{G}(a, b)}+\sum_{a \in V\left(G_{1}\right)} \sum_{b \in V\left(G_{2}\right)} \frac{1}{r_{G}(a, b)} \\
= & \operatorname{RH}\left(G_{1}\right)+\operatorname{RH}\left(G_{2}\right) \\
& +\sum_{a \in V\left(G_{1}\right)} \sum_{b \in V\left(G_{2}\right)} \frac{1}{r_{G}(a, x)+r_{G}(b, y)+1} .
\end{aligned}
$$

This completes the proof. 


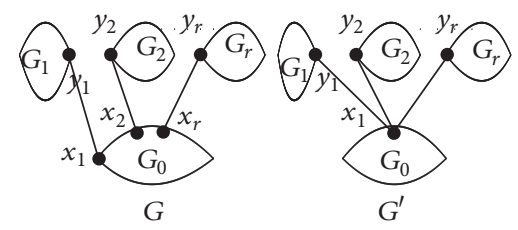

FIgURE 1: The graphs in Lemma 5.
Lemma 5. Let $G$ and $G^{\prime}$ be the graphs in Figure 1 , where $G_{0}$ is a complete graph, and then $R H(G) \leq R H\left(G^{\prime}\right)$, with equality if and only if $x_{1}=x_{2}=\cdots=x_{r}$.

Proof. From the definition of the Resistance-Harary index and Lemma 4 , we have

$$
\begin{aligned}
\mathrm{RH}(G)= & \sum_{1 \leq i<j \leq n} \frac{1}{r_{G}\left(v_{i}, v_{j}\right)}=\sum_{i=0}^{r} \mathrm{RH}\left(G_{i}\right)+\sum_{i=1}^{r} \sum_{a \in V\left(G_{0}\right)} \sum_{b \in V\left(G_{i}\right)} \frac{1}{r_{G}(a, b)}+\sum_{1 \leq i<j \leq r} \sum_{b \in V\left(G_{i}\right)} \sum_{c \in V\left(G_{j}\right)} \frac{1}{r_{G}(b, c)} \\
= & \sum_{i=0}^{r} \mathrm{RH}\left(G_{i}\right)+\sum_{i=1}^{r} \sum_{a \in V\left(G_{0}\right)} \sum_{b \in V\left(G_{i}\right)} \frac{1}{r_{G_{0}}\left(a, x_{i}\right)+r_{G_{i}}\left(y_{i}, b\right)+1} \\
& +\sum_{1 \leq i<j \leq r} \sum_{b \in V\left(G_{i}\right)} \sum_{c \in V\left(G_{j}\right)} \frac{1}{r_{G_{i}}\left(b, y_{i}\right)+r_{G_{j}}\left(c, y_{j}\right)+2+r_{G_{0}}\left(x_{i}, x_{j}\right)} .
\end{aligned}
$$

Similarly,

$$
\begin{aligned}
\mathrm{RH} & \left(G^{\prime}\right)=\sum_{1 \leq i<j \leq n} \frac{1}{r_{G^{\prime}}\left(v_{i}, v_{j}\right)} \\
= & \sum_{i=0}^{r} \mathrm{RH}\left(G_{i}\right)+\sum_{i=1}^{r} \sum_{a \in V\left(G_{0}\right)} \sum_{b \in V\left(G_{i}\right)} \frac{1}{r_{G^{\prime}}(a, b)} \\
& +\sum_{1 \leq i<j \leq r} \sum_{b \in V\left(G_{i}\right)} \sum_{c \in V\left(G_{j}\right)} \frac{1}{r_{G^{\prime}}(b, c)}
\end{aligned}
$$

$$
\begin{aligned}
= & \sum_{i=0}^{r} \operatorname{RH}\left(G_{i}\right) \\
& +\sum_{i=1}^{r} \sum_{a \in V\left(G_{0}\right)} \sum_{b \in V\left(G_{i}\right)} \frac{1}{r_{G_{0}}\left(a, x_{1}\right)+r_{G_{i}}\left(y_{i}, b\right)+1} \\
& +\sum_{1 \leq i<j \leq r} \sum_{b \in V\left(G_{i}\right)} \sum_{c \in V\left(G_{j}\right)} \frac{1}{r_{G_{i}}\left(b, y_{i}\right)+r_{G_{j}}\left(c, y_{j}\right)+2} .
\end{aligned}
$$

Since $G_{0}$ is a complete graph, $r_{G_{0}}\left(a, x_{1}\right)=r_{G_{0}}\left(a, x_{i}\right)=2 / n_{0}$ for $1 \leq i \leq r$. And

$$
\mathrm{RH}\left(G^{\prime}\right)-\mathrm{RH}(G)=\sum_{1 \leq i<j \leq r} \sum_{b \in V\left(G_{i}\right)} \sum_{c \in V\left(G_{j}\right)}\left(\frac{1}{r_{G_{i}}\left(b, y_{i}\right)+r_{G_{j}}\left(c, y_{j}\right)+2}-\frac{1}{r_{G_{i}}\left(b, y_{i}\right)+r_{G_{j}}\left(c, y_{j}\right)+2+r_{G_{0}}\left(x_{i}, x_{j}\right)}\right) \geq 0
$$

with equality if and only if $r_{G_{0}}\left(x_{i}, x_{j}\right)=0$; that is, $x_{1}=x_{2}=$ $\cdots=x_{r}$. This completes the proof.

Lemma 6. Let $G$ and $G^{\prime}$ be the graphs depicted in Figure 2, where $G_{2}$ and $G_{3}$ are all complete graphs. Let $n_{i}$ be the number of vertices of $G_{i}$, where $i=2,3$. If $n_{3}>n_{2}$ and $V\left(G_{4}\right)$ may be an empty set, then $R H\left(G^{\prime}\right)>R H(G)$.

Proof. By the definition of the Resistance-Harary index and Lemmas 1 and 4, we have

$$
\mathrm{RH}(G)=\sum_{1 \leq i<j \leq n} \frac{1}{r_{G}\left(v_{i}, v_{j}\right)}
$$

$$
\begin{aligned}
= & \sum_{i=1}^{4} \mathrm{RH}\left(G_{i}\right)+\sum_{i=2}^{4} \sum_{a \in V\left(G_{1}\right)} \sum_{b \in V\left(G_{i}\right)} \frac{1}{r_{G}(a, b)} \\
& +\sum_{2 \leq i<j \leq 4} \sum_{a \in V\left(G_{i}\right)} \sum_{b \in V\left(G_{j}\right)} \frac{1}{r_{G}(a, b)} .
\end{aligned}
$$

Since $G_{2}$ and $G_{3}$ are all complete graphs, for any two vertices $x, y \in V\left(G_{i}\right)$, we have $r_{G_{i}}(x, y)=2 / n_{i}$, where $i=2,3$. Similarly, we have 


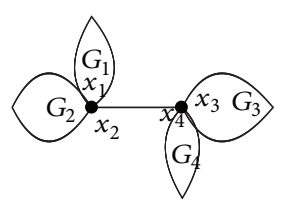

G

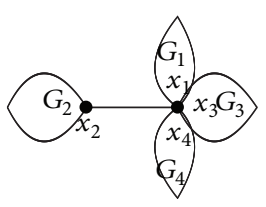

$G^{\prime}$

FIgURE 2: The graphs in Lemma 6.

$$
\begin{aligned}
\mathrm{RH}\left(G^{\prime}\right)= & \sum_{1 \leq i<j \leq n} \frac{1}{r_{G^{\prime}}\left(v_{i}, v_{j}\right)}=\sum_{i=1}^{4} \mathrm{RH}\left(G_{i}\right)+\sum_{i=2}^{4} \sum_{a \in V\left(G_{1}\right)} \sum_{b \in V\left(G_{i}\right)} \frac{1}{r_{G^{\prime}}(a, b)}+\sum_{2 \leq i<j \leq 4} \sum_{a \in V\left(G_{i}\right)} \sum_{b \in V\left(G_{j}\right)} \frac{1}{r_{G^{\prime}}(a, b)}, \\
\mathrm{RH}\left(G^{\prime}\right)-\mathrm{RH}(G)= & \sum_{a \in V\left(G_{1}\right)} \sum_{b \in V\left(G_{3}\right)}\left\{\frac{1}{r_{G_{1}}\left(a, x_{1}\right)+r_{G_{3}}\left(b, x_{3}\right)}-\frac{1}{r_{G_{1}}\left(a, x_{1}\right)+r_{G_{3}}\left(b, x_{3}\right)+1}\right\} \\
& -\sum_{a \in V\left(G_{1}\right)} \sum_{b \in V\left(G_{2}\right)}\left\{\frac{1}{r_{G_{1}}\left(a, x_{1}\right)+r_{G_{2}}\left(b, x_{2}\right)}-\frac{1}{r_{G_{1}}\left(a, x_{1}\right)+r_{G_{2}}\left(b, x_{2}\right)+1}\right\} \\
& +\sum_{a \in V\left(G_{1}\right)} \sum_{b \in V\left(G_{4}\right)}\left\{\frac{1}{r_{G_{1}}\left(a, x_{1}\right)+r_{G_{4}}\left(b, x_{4}\right)}-\frac{1}{r_{G_{1}}\left(a, x_{1}\right)+r_{G_{4}}\left(b, x_{4}\right)+1}\right\} .
\end{aligned}
$$

We just need to prove that

$$
\begin{aligned}
\nabla_{1}= & \sum_{a \in V\left(G_{1}\right)} \sum_{b \in V\left(G_{3}\right)}\left\{\frac{1}{r_{G_{1}}\left(a, x_{1}\right)+r_{G_{3}}\left(b, x_{3}\right)}-\frac{1}{r_{G_{1}}\left(a, x_{1}\right)+r_{G_{3}}\left(b, x_{3}\right)+1}\right\} \\
& -\sum_{a \in V\left(G_{1}\right)} \sum_{b \in V\left(G_{2}\right)}\left\{\frac{1}{r_{G_{1}}\left(a, x_{1}\right)+r_{G_{2}}\left(b, x_{2}\right)}-\frac{1}{r_{G_{1}}\left(a, x_{1}\right)+r_{G_{2}}\left(b, x_{2}\right)+1}\right\}>0 .
\end{aligned}
$$

In fact,

$$
\nabla_{1}=\sum_{a \in V\left(G_{1}\right)}\left\{\frac{n_{3}}{\left(r_{G_{1}}\left(a, x_{1}\right)+2 / n_{3}\right)\left(r_{G_{1}}\left(a, x_{1}\right)+2 / n_{3}+1\right)}-\frac{n_{2}}{\left(r_{G_{1}}\left(a, x_{1}\right)+2 / n_{2}\right)\left(r_{G_{1}}\left(a, x_{1}\right)+2 / n_{2}+1\right)}\right\} .
$$

In the following, we just need to prove that the fraction of $\nabla_{1}$ is greater than zero. Denote $\nabla_{2}$ to be the fraction of $\nabla_{1}$; in fact,

$$
\begin{aligned}
\nabla_{2}= & \left(r_{G_{1}}\left(a, x_{1}\right)\right)^{2}\left(n_{3}-n_{2}\right) \\
& +r_{G_{1}}\left(a, x_{1}\right)\left[\frac{n_{3}\left(4+n_{2}\right)}{n_{2}}-\frac{\left(4+n_{3}\right) n_{2}}{n_{3}}\right] \\
& +\frac{2 n_{3}\left(2+n_{2}\right)}{n_{2}^{2}}-\frac{2 n_{2}\left(n_{3}+2\right)}{n_{3}^{2}},
\end{aligned}
$$
Since $n_{3}>n_{2}, \operatorname{RH}\left(G^{\prime}\right)>\operatorname{RH}(G)$. This completes the proof. 

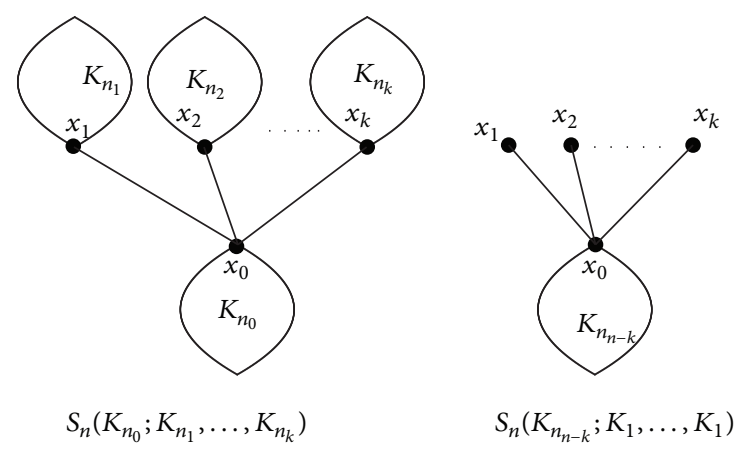

Figure 3: The graphs in Lemma 7 and in Theorem 9.

\section{Characterization of the Maximizing Graph}

In this section, we will characterize maximizing graph among all connected graphs with $n$ vertices and $k$ cut edges.

First, we need some definitions below; let $s_{k+1}$ be a star with vertex set $\left\{v_{0}, v_{1}, \ldots, v_{k}\right\}$, where $v_{0}$ is the center of the star. In particular, the graph $S_{n}\left(K_{n_{0}} ; K_{n_{1}}, \ldots, K_{n_{k}}\right)$ with $\sum_{i=0}^{k} n_{i}=n$ is obtained from $S_{k+1}$ by replacing the vertex $v_{i}$ by $K_{n_{i}}$ for $i=0,1,2, \ldots, k$. By the definition of ResistanceHarary index, it is not difficult to obtain $\mathrm{RH}\left(K_{n}\right)=n^{2}(n-$ 1)/4. If $G_{0}$ is a complete graph, for any vertices $x_{0}, a \in V\left(G_{0}\right)$, there is $r_{G_{0}}\left(a, x_{0}\right)=2 / n_{0}$.

Lemma 7. Let $n$ and $n_{0}, n_{1}, \ldots, n_{k}(k \geq 1)$ be positive integers such that $n_{0} \geq n_{1} \geq \cdots \geq n_{k}, n_{1}>1$, and $n_{0}+n_{1}+\cdots+$ $n_{k}=n$. Let $G=S_{n}\left(K_{n_{0}}, K_{n_{1}}, \ldots, K_{n_{k}}\right)$ (see Figure 3$)$ and $G^{\prime}=$ $S_{n}\left(K_{n_{0}+1}, K_{n_{1}-1}, \ldots, K_{n_{k}}\right)$. Then $R H\left(G^{\prime}\right)>R H(G)$.
Proof. By direct calculation, we have

$$
\begin{aligned}
\mathrm{RH} & (G)=\mathrm{RH}\left(S_{n}\left(K_{n_{0}} ; K_{n_{1}}, \ldots, K_{n_{k}}\right)\right) \\
= & \sum_{i=0}^{k} \mathrm{RH}\left(K_{n_{i}}\right)+\sum_{i=1}^{k} \sum_{a \in V\left(K_{n_{0}}\right)} \sum_{b \in V\left(K_{n_{i}}\right)} \frac{1}{r_{G}(a, b)} \\
& +\sum_{1 \leq i<j \leq k} \sum_{b \in V\left(K_{n_{i}}\right)} \sum_{c \in V\left(K_{n_{j}}\right)} \frac{1}{r_{G}(b, c)} \\
= & \frac{1}{4} \sum_{i=0}^{k} n_{i}^{2}\left(n_{\mathrm{i}}-1\right) \\
& +\sum_{i=1}^{k} \sum_{a \in V\left(K_{n_{0}}\right)} \sum_{b \in V\left(K_{n_{i}}\right)} \frac{1}{r_{G}\left(a, x_{0}\right)+r_{G}\left(b, x_{i}\right)+1} \\
& +\sum_{1 \leq i<j \leq k} \sum_{b \in V\left(K_{n_{i}}\right)} \sum_{c \in V\left(K_{n_{j}}\right)} \frac{1}{r_{G}\left(b, x_{i}\right)+r_{G}\left(c, x_{j}\right)+2} \\
= & \frac{1}{4} \sum_{i=0}^{k} n_{i}^{2}\left(n_{i}-1\right)+\sum_{i=1}^{k} \frac{n_{0}}{2 / n_{0}+2 / n_{i}+1} \\
& +\sum_{1 \leq i<j \leq k} \sum_{b \in V\left(K_{n_{i}}\right)} \sum_{c \in V\left(K_{n_{j}}\right)} \frac{1}{2 / n_{i}+2 / n_{j}+2} .
\end{aligned}
$$

Similarly, we can deduce the value of $\operatorname{RH}\left(G^{\prime}\right)$. Then

$$
\begin{aligned}
\mathrm{RH}\left(G^{\prime}\right)-\mathrm{RH}(G)= & \frac{1}{4}\left\{\left(n_{0}+1\right)^{2} n_{0}-n_{0}^{2}\left(n_{0}-1\right)+\left(n_{1}-1\right)^{2}\left(n_{1}-2\right)-n_{1}^{2}\left(n_{1}-1\right)\right\} \\
& +\left\{\frac{n_{0}+1}{2 /\left(n_{0}+1\right)+2 /\left(n_{1}-1\right)+1}-\frac{n_{0}}{2 / n_{0}+2 / n_{1}+1}\right\} \\
& +\sum_{i=2}^{k}\left\{\frac{n_{0}+1}{2 /\left(n_{0}+1\right)+2 / n_{i}+1}-\frac{n_{0}}{2 / n_{0}+2 / n_{i}+1}\right\} \\
& +\sum_{i=2}^{k}\left\{\frac{n_{1}-1}{2 /\left(n_{1}-1\right)+2 / n_{i}+2}-\frac{n_{1}}{2 / n_{1}+2 / n_{i}+2}\right\} .
\end{aligned}
$$

In order to prove that the result $\mathrm{RH}\left(G^{\prime}\right)>\operatorname{RH}(G)$, we

distinguish three steps in the following. Denote

$$
\begin{aligned}
& \triangle_{1}=\frac{1}{4}\left\{\left(n_{0}+1\right)^{2} n_{0}-n_{0}^{2}\left(n_{0}-1\right)+\left(n_{1}-1\right)^{2}\left(n_{1}-2\right)-n_{1}^{2}\left(n_{1}-1\right)\right\}-\frac{n_{0}}{2 / n_{0}+2 / n_{1}+1}, \\
& \triangle_{2}=\frac{n_{0}+1}{2 /\left(n_{0}+1\right)+2 /\left(n_{1}-1\right)+1}, \\
& \triangle_{3}=\sum_{i=2}^{k}\left\{\left(\frac{n_{0}+1}{2 /\left(n_{0}+1\right)+2 / n_{i}+1}-\frac{n_{0}}{2 / n_{0}+2 / n_{i}+1}\right)+\left(\frac{n_{1}-1}{2 /\left(n_{1}-1\right)+2 / n_{i}+2}-\frac{n_{1}}{2 / n_{1}+2 / n_{i}+2}\right)\right\} .
\end{aligned}
$$


Obviously, $\Delta_{2}>0$. In the following, we first prove that $\triangle_{1}>0$. By direct calculation, we have

$$
\begin{aligned}
\triangle_{1}= & \frac{1}{4}\left\{3 n_{0}^{2}+n_{0}-3 n_{1}^{2}+5 n_{1}-2\right\} \\
& -\frac{n_{0}}{2 / n_{0}+2 / n_{1}+1} \\
= & \frac{n_{1}-1}{2}+\frac{3 n_{0}^{2}-3 n_{1}^{2}+n_{0}+3 n_{1}}{4}
\end{aligned}
$$

$$
\begin{aligned}
& -\frac{n_{0}^{2} n_{1}}{2 n_{1}+2 n_{0}+n_{0} n_{1}} \\
= & \frac{n_{1}-1}{2}+\frac{3 n_{0} n_{1}\left(n_{0}-n_{1}\right)+3 n_{0} n_{1}\left(n_{0}^{2}-n_{1}^{2}\right)}{4\left(2 n_{1}+2 n_{0}+n_{0} n_{1}\right)} \\
& +\frac{6\left(n_{0}^{3}-n_{1}^{3}\right)+\left(8 n_{0} n_{1}+2 n_{0}^{2}+6 n_{1}^{2}\right)}{4\left(2 n_{1}+2 n_{0}+n_{0} n_{1}\right)}>0
\end{aligned}
$$

(since $\left.n_{0} \geq n_{1}>1\right)$. Secondly, we will prove that $\triangle_{3}>0$.

$$
\begin{aligned}
\triangle_{3} & =\sum_{i=2}^{k}\left\{\left(\frac{n_{0}+1}{2 /\left(n_{0}+1\right)+2 / n_{i}+1}-\frac{n_{0}}{2 / n_{0}+2 / n_{i}+1}\right)-\left(\frac{n_{1}}{2 / n_{1}+2 / n_{i}+2}-\frac{n_{1}-1}{2 /\left(n_{1}-1\right)+2 / n_{i}+2}\right)\right\} \\
& >\sum_{i=2}^{k}\left\{\left(\frac{n_{0}+1}{2 /\left(n_{0}+1\right)+2 / n_{i}+1}-\frac{n_{0}}{2 / n_{0}+2 / n_{i}+1}\right)-\left(\frac{n_{1}}{2 / n_{1}+2 / n_{i}+1}-\frac{n_{1}-1}{2 /\left(n_{1}-1\right)+2 / n_{i}+1}\right)\right\}>0
\end{aligned}
$$

where the second inequality is due to the fact that

$$
\begin{aligned}
& \frac{n_{1}}{2 / n_{1}+2 / n_{i}+1}-\frac{n_{1}-1}{2 /\left(n_{1}-1\right)+2 / n_{i}+1} \\
& -\frac{n_{1}}{2 / n_{1}+2 / n_{i}+2}-\frac{n_{1}-1}{2 /\left(n_{1}-1\right)+2 / n_{i}+2} \\
& =\frac{n_{1}}{\left(2 / n_{1}+A\right)\left(2 / n_{1}+B\right)} \\
& -\frac{n_{1}-1}{\left(2 /\left(n_{1}-1\right)+B\right)\left(2 /\left(n_{1}-1\right)+A\right)},
\end{aligned}
$$

where $A=2 / n_{i}+1, B=2 / n_{i}+2$, and the function $f(x)=$ $x /(2 / x+A)(2 / x+B)$ is an increasing function, and then

$$
\begin{gathered}
\frac{n_{1}}{2 / n_{1}-2 / n_{i}+1}-\frac{n_{1}-1}{2 /\left(n_{1}-1\right)+2 / n_{i}+1} \\
>\frac{n_{1}}{2 / n_{1}+2 / n_{i}+2}-\frac{n_{1}-1}{2 /\left(n_{1}-1\right)+2 / n_{i}+2} .
\end{gathered}
$$

By Lemma 2, the last inequality is due to the fact the function $f_{2}(x)=x /(2 / x+A)-(x-1) /(2 /(x-1)+A)$ for $x \geq 2$ and $0<A \leq 3$ is an increasing function.

By combination with above discussion, we have $\mathrm{RH}\left(G^{\prime}\right)>$ $\mathrm{RH}(G)$, which finishes the proof of Lemma 7 .

Corollary 8. Suppose that $R H\left(S_{n}\left(K_{n_{0}} ; K_{n_{1}}, \ldots, K_{n_{k}}\right)\right)$ is defined as above and $n_{0} \geq n_{1} \geq n_{2} \geq \cdots \geq n_{k}$, the value of $R H\left(S_{n}\left(K_{n_{0}} ; K_{n_{1}}, \ldots, K_{n_{k}}\right)\right)$ reaches its maximum value at $n_{0}=n-k$ and $n_{1}=n_{2}=\cdots=n_{k}=1$.
Theorem 9. If $G$ is a connected graph with $k$ cut edges and $n$ vertices, then

$$
\begin{aligned}
R H(G) \leq & \frac{(n-k)^{2}(n-k-1)}{4}+\frac{k^{2}+3 k}{4} \\
& +\frac{(n-k-1)(n-k) k}{n-k+2},
\end{aligned}
$$

with equality if and only if $G \cong S_{n}\left(K_{n-k} ; K_{1}, K_{1}, \ldots, K_{1}\right)$ (see Figure 3).

Proof. To determine the maximum Resistance-Harary index of the graph, we select such a connected graph so that it cut off all the cut edges for a complete graph by Lemma 3. Moreover, we can further choose $G=S_{n}\left(K_{n_{0}} ; K_{n_{1}}, \ldots, K_{n_{k}}\right)$ by Lemmas 5 and 6 , and let $\sum_{i=0}^{k} n_{i}=n, n_{0}=\max \left\{n_{0}, n_{1}, \ldots, n_{k}\right\}$. From the definition of the Resistance-Harary index and Lemma 4, we have

$$
\begin{aligned}
\mathrm{RH} & (G)=\mathrm{RH}\left(S_{n}\left(K_{n_{0}} ; K_{n_{1}}, \ldots, K_{n_{k}}\right)\right) \\
= & \frac{1}{4} \sum_{i=0}^{k} n_{i}^{2}\left(n_{i}-1\right) \\
& +\sum_{i=1}^{k} \sum_{a \in V\left(K_{n_{0}}\right)} \sum_{b \in V\left(K_{n_{i}}\right)} \frac{1}{r_{G}\left(a, x_{0}\right)+r_{G}\left(b, x_{i}\right)+1} \\
& +\sum_{1 \leq i<j \leq k} \sum_{b \in V\left(K_{n_{i}}\right)} \sum_{c \in V\left(K_{n_{j}}\right)} \frac{1}{r_{G}\left(b, x_{i}\right)+r_{G}\left(c, x_{j}\right)+2} \\
= & \sum_{i=0}^{k} n_{i}^{2}\left(n_{i}-1\right)+\sum_{i=1}^{k} \frac{n_{0}}{2 / n_{0}+2 / n_{j}+1} \\
& +\sum_{1 \leq i<j \leq k} \sum_{b \in V\left(K_{n_{i}}\right)} \sum_{c \in V\left(K_{n_{j}}\right)} \frac{1}{2 / n_{i}+2 / n_{j}+2}
\end{aligned}
$$




$$
\begin{aligned}
\leq & \frac{(n-k)^{2}(n-k-1)}{4}+\frac{k^{2}+3 k}{4} \\
& +\frac{k(n-k)(n-k-1)}{n-k+2} .
\end{aligned}
$$

By Lemma 7 and Corollary 8, we know that the equality holds if and only if $n_{0}=n-k, n_{1}=n_{2}=\cdots=n_{k}=1$; that is, $G \cong S_{n}\left(K_{n-k} ; K_{1}, \ldots, K_{1}\right)$. This completes the result.

Corollary 10. If $T$ is a tree with $n$ vertices, then $R H(G) \leq\left(n^{2}+\right.$ $n-2) / 4$, with equality if and only if $T \cong S_{n}$.

Proof. Since $T$ is a tree with $n$ vertices, it has $n-1$ cut edges, and $\operatorname{RH}(G) \leq\left(n^{2}+n-2\right) / 4$ with equality if and only if $T \cong S_{n}$ by Theorem 9 . This completes the result.

\section{Conclusion}

In this thesis, we considered the Resistance-Harary index of graphs with given number of cut edges and describe a graph with a maximum Resistance-Harary index. A problem raised naturally at this moment is, among all connected graphs with $n$ vertices and $k$ cut edges, which graph has the minimum Resistance-Harary index? We will continue to consider this problem in the nearest future.

\section{Conflicts of Interest}

The authors declare no competing financial interests.

\section{Acknowledgments}

Wang was supported by National Natural Science Foundation of China under Grant no. 11571135 and Humanities and Social Sciences of Ministry of Education Planning Fund under Grant no. 16YJA630032. Hua was supported in part by National Natural Science Foundation of China under Grant no. 11571135 .

\section{References}

[1] D. J. Klein and M. Randić, "Resistance distance," Journal of Mathematical Chemistry, vol. 12, no. 1-4, pp. 81-95, 1993.

[2] D. J. Klein, "Graph geometry, graph metrices and Wiener," MATCH-Communications in Mathematical and in Computer Chemistry, vol. 35, pp. 7-27, 1997.

[3] D. J. Klein and H.-Y. Zhu, "Distances and volumina for graphs," Journal of Mathematical Chemistry, vol. 23, no. 1-2, pp. 179-195, 1998.

[4] D. Plavšić, S. Nikolić, N. Trinajstić, and Z. Mihalić, "On the Harary index for the characterization of chemical graphs," Journal of Mathematical Chemistry, vol. 12, no. 1-4, pp. 235-250, 1993.

[5] O. Ivanciuc, T.-S. Balaban, and A. T. Balaban, "Design of topological indices. Part 4. Reciprocal distance matrix, related local vertex invariants and topological indices," Journal of Mathematical Chemistry, vol. 12, no. 1-4, pp. 309-318, 1993.

[6] I. Gutman, "A property of Wiener number and its modifications," Indian Journal of Chemistry, vol. 36, pp. 128-132, 1997.
[7] K. Xu, "Trees with the seven smallest and eight greatest Harary indices," Discrete Applied Mathematics, vol. 160, no. 3, pp. 321331, 2012.

[8] K. Xu, K. C. Das, and N. Trinajstić, The Harary Index of a Graph, Springer, New York, NY, USA, 2014.

[9] D. J. Klein and O. Ivanciuc, "Graph cyclicity, excess conductance, and resistance deficit," Journal of Mathematical Chemistry, vol. 30, no. 3, pp. 271-287, 2001.

[10] Y. Yang, "On a new cyclicity measure of graphs: the global cyclicity index," Discrete Applied Mathematics, vol. 172, pp. 8897, 2014.

[11] S. Chen, Z. Guo, T. Zeng, and L. Yang, "On the resistanceHarary index of unicyclic graphs," MATCH-Communications in Mathematical and in Computer Chemistry, vol. 78, no. 1, pp. 189-198, 2017.

[12] J. A. Bondy and U. S. R. Murty, Graph Theory with Application, Macmillan Press, New York, NY, USA, 1976.

[13] H. Deng, "On the minimum Kirchhoff index of graphs with a given number of cut-edges," MATCH-Communications in Mathematical and in Computer Chemistry, vol. 63, no. 1, pp. 171180, 2010.

[14] H. Wang, H. Hua, and D. Wang, "Cacti with minimum, secondminimum, and third-minimum Kirchhoff indices," Mathematical Communications, vol. 15, no. 2, pp. 347-358, 2010. 

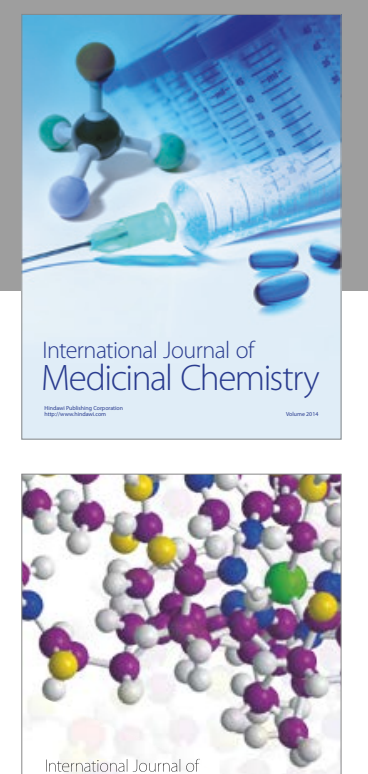

Carbohydrate Chemistry

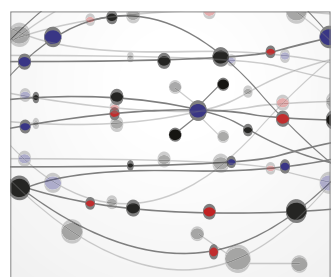

The Scientific World Journal
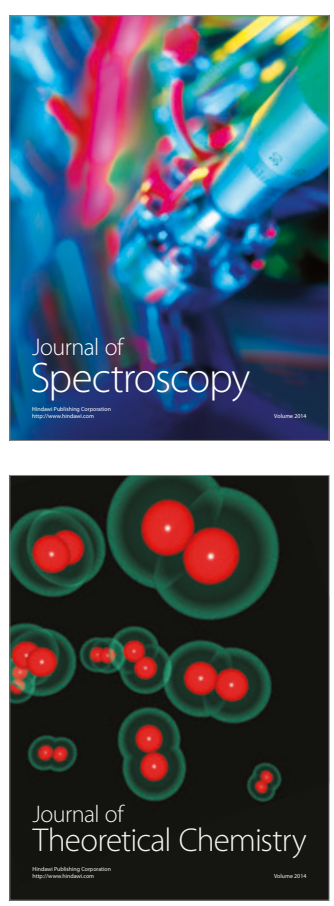
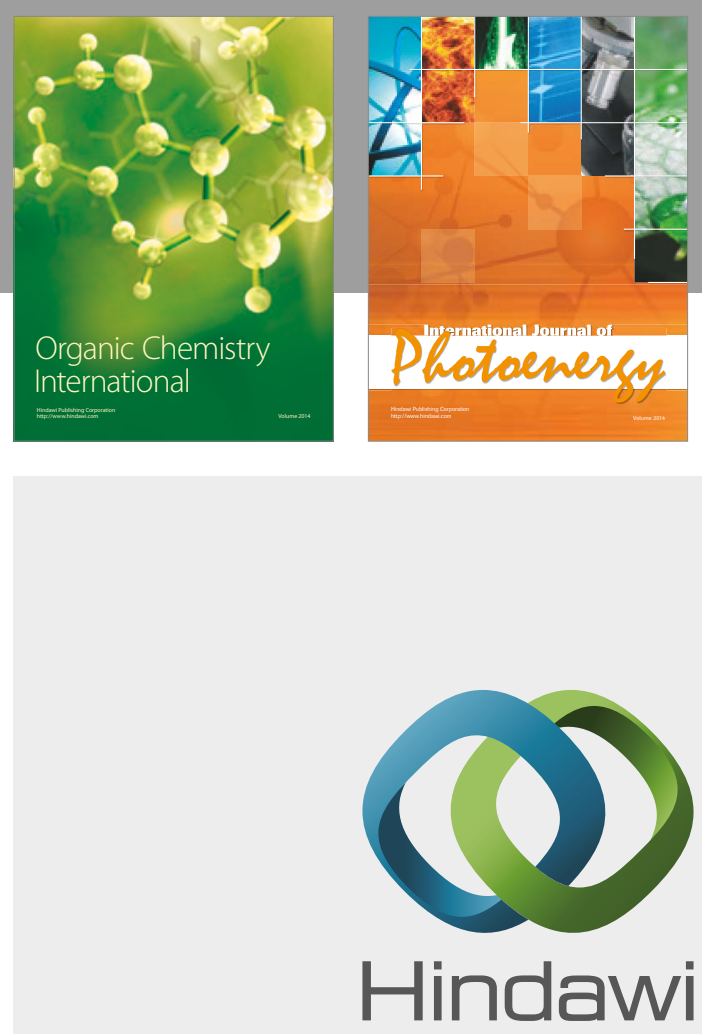

Submit your manuscripts at

https://www.hindawi.com

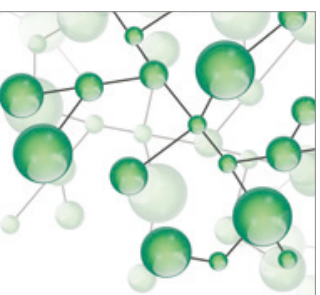

International Journal of

Inorganic Chemistry

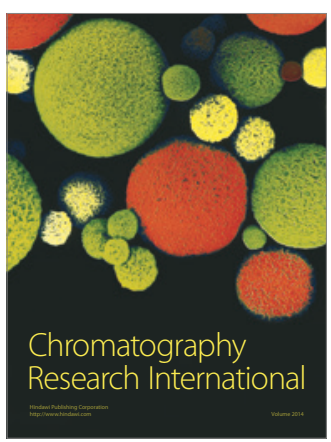

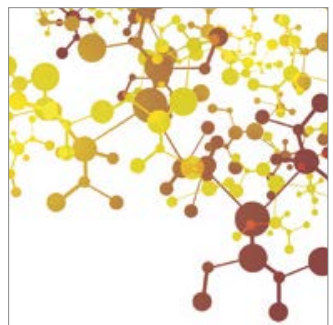

Applied Chemistry
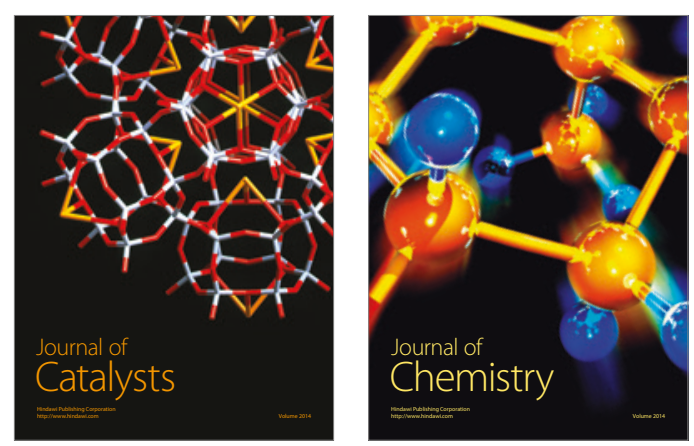
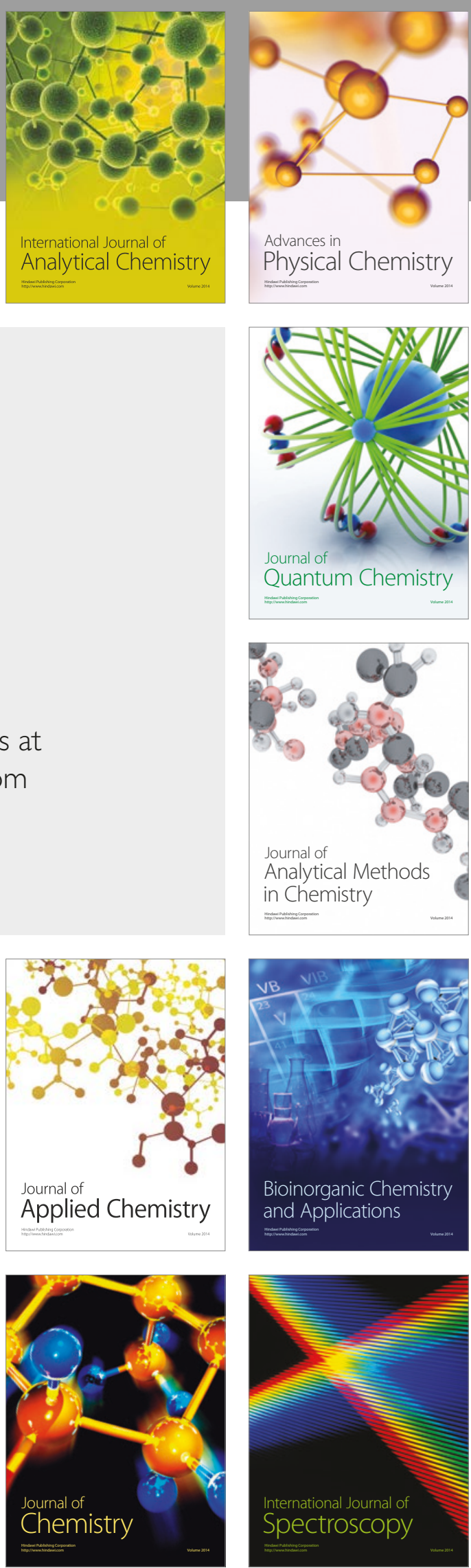